\title{
LTE Transmission Exploiting Pulse Width Modulation in Fibre Optic Links
}

\author{
P. Parolari, A. Gatto, L. Combi, R. Sacchi*, R. Giacometti*, A. Lena*, C. Canziani*, \\ and U. Spagnolini, Senior Member, IEEE \\ Politecnico di Milano, Dip. Elettronica Informazione e Bioingegneria, Via Ponzio 34/5,20133 Milano, Italy \\ "Keysight Technologies Italy Srl, via P.Gobetti 2/C, 20063 Cernusco sul Naviglio (MI), Italy \\ Tel: +390223998926, e-mail: paola.parolari@polimi.it
}

\begin{abstract}
Recently it has been demonstrated that pulse width modulation (PWM) can represent a viable solution for the analog optical fronthaul alternative to standard radio over fibre, which still avoids the bandwidth expansion of the digital fronthaul. The PWM encodes the analog samples at the transmitter onto the duration of the on/off keyed optical signal, splitting the sampling and quantization of the radio signal between remote radio units (RRUs) and baseband units (BBUs). In particular in this contribution we demonstrate the capabilities of optical PWM for the transport of LTE signals to support the centralized access network (C-RAN) fronthaul in fibre optic link up to $10-\mathrm{km}$ of standard single mode fibre. The generation and analysis of the radio signals is provided by software modules compliant with the LTE standard which allowed to analyse performance results for the different LTE carriers, channels and services. The PWM optical signal connecting RRUs to BBUs is generated by either directly modulating a DFB laser or an externally seeded reflective semiconductor optical amplifier (RSOA). Both devices could be exploited inside a wavelength division multiplexed passive optical network (WDM PON) architecture where the various RRU-to-BBU links are pooled through virtual point-to-point connections at different wavelengths.
\end{abstract}

Keywords: WDM PON, fronthaul, PWM, RSOA, external-seeding.

\section{INTRODUCTION}

Next generation (LTE and 5G) mobile communication networks are expected to cope with the ever-growing demand for high throughput, mobility and low latency which are changing the radio access networks (RANs) paradigm toward the centralized RAN (C-RAN). This architecture should be able to handle the growing demand of capacity and the increasing pervasivity of cellular systems. The C-RAN can be designed to support a large number of users taking advantage of mutual cooperation for interference mitigation and of a huge number of antennas for massive MIMO deployment [1]. In C-RANs the antennas, together with all the RF functionalities, are hosted at the cell sites in the remote radio heads (RRHs), which need to forward the signal to/from the BBUs through the fronthaul (FH) link. Due to the high available capacity preferred FH links exploit fiber optic transmission based on the common public radio interface (CPRI) protocol [2,3]. However digital FH leads to a bandwidth expansion not sustainable for next RAN requirements. Although CPRI compression or different RAN functional splits have been proposed a full-analog transmission, enabling the reduction of the bandwidth requirements, the latency and the hardware costs, will likely outperform other solutions. Recent strategies adopt multiple frequency-domain multiplexed (FDM) LTE signals assisted by analog and digital signal processing [4] or directly-modulated multiple intermediate frequency-over-fiber (IFoF) schemes [5]. On the other hand, analog radio-over-fiber (RoF) suffers from linear and nonlinear distortions (NLD) due to laser diode inherent nonlinearities, chromatic dispersion, fiber nonlinear impairments and from limited power budget.

We have recently demonstrated [6] that optical pulse-width modulation (PWM) can represent an alternative analog coding to overcome some of the previously evidenced RoF limitations. The PWM in fact is a non-linear modulation that encodes the analog samples at the transmitter (e.g., at the RRU) onto the duration of the on/off keyed optical signal, with several benefits in terms of simplicity and tolerance towards NLD [7]. In particular in this contribution we demonstrate the capabilities of optical PWM for the transport of LTE signals with variable bandwidths (e.g. $3 \mathrm{MHz}$ and $20 \mathrm{MHz}$ ) over fibre optic links up to 10-km of standard single mode fibre (SSMF). The generation and analysis of the radio signals is provided by software modules compliant with the LTE standard which allowed to analyse performance results for the different LTE carriers, channels and services. The PWM optical signal connecting RRUs to BBUs is generated by either directly modulating a DFB laser or an externally seeded reflective semiconductor optical amplifier (RSOA). Both devices could be exploited inside a wavelength division multiplexed passive optical network (WDM PON) architecture where the various RRU-toBBU links are pooled through virtual point-to-point connections at different wavelengths. 


\section{OPTICAL PWM ARCHITECTURE AND EXPERIMENTAL SET UP}

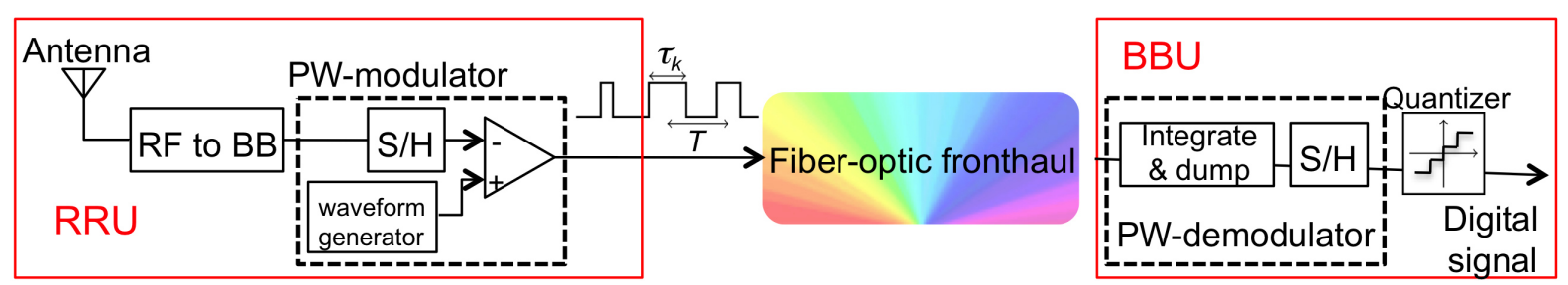

Figure 1. PWM modulator and demodulator.

Figure 1 shows the proposed PWM architecture, in which the sampling and quantization of the baseband signal are split between the RRU and the BBU. The FH link joining the RRUs and BBUs is performed encoding and transmitting IQ signals by the optical PWM over fibre optic connections. This approach avoids the bandwidth expansion of digital IQ streaming due to the quantization and can support multiple RRU-to-BBU links with multiple fibers or thanks to the exploitation of wavelength division multiplexing in PONs. Focusing on upstream transmission (RRU-to-BBU) in Fig. 1a), the RF signal is down-converted and it is harmonically sampled by the PW modulator [8]. The samples of the I and Q components are transmitted to the BBU via the fronthaul fiber optic network as variable-duration on-off keyed optical signals: every pulse of duration $\tau_{\mathrm{k}}$ belongs to one interval $\mathrm{T}\left(\tau_{\mathrm{k}}<\mathrm{T}\right)$. At the BBU site, after detection, the demodulator transforms the PW signals into amplitudes by an integrator that is periodically reset every interval $\mathrm{T}$. The interval $\mathrm{T}$ is chosen according to the mobile signal. For the employed LTE signals (3-MHz and 20-MHz bandwidth) T is set respectively to $167 \mathrm{~ns}$ and $25 \mathrm{~ns}$ [9].

Figure 2 shows the employed experimental set up. At the transmitter we exploited the SystemVue ${ }^{\circledR}$ environment to generate a LTE signal; the IQ samples to be transmitted are fed to a Matlab ${ }^{\circledR}$ algorithm that provides the amplitude-to-duration mapping to perform the PWM. The real and the imaginary part of the IQ samples are coded onto two durations which are output as two rectangular PWM pulses. The PWM signal is sampled and played at $65 \mathrm{GS} / \mathrm{s}$ by an arbitrary waveform generator (Keysight M8195A), which drives an E/O converter. The E/O converter is either a $15-\mathrm{GHz}$ directly modulated laser (DML) at 1550-nm or a C-band RSOA with $3-\mathrm{GHz}$ E/O bandwidth. In this latter case the RSOA is externally seeded by a DFB laser at $1552 \mathrm{~nm}$ located at the receiver side in order to test a colourless WDM PON architecture [10]. Two WDM multiplexer/ demultiplexers and $10 \mathrm{~km}$ of SSMF constitute the optical distribution network (ODN), moreover a variable optical attenuator (VOA) allows to vary overall ODN losses. The main differences in the transmitters is represented by the relative intensity noise (RIN) associated to the E/O converter which is slightly higher for the externally seeded RSOA [11]. After propagation, the PWM signal is detected by a 1-GHz PIN photodiode. The received signal is fed to a 33-GHz 80-GS/s digital storage oscilloscope (Keysight V334A); a Matlab® algorithm estimates the pulse widths and performs the duration-to-amplitude mapping obtaining the received IQ samples. Both transmitted and received IQ samples are fed to the $89601 \mathrm{~B}$ vector signal analyzer (VSA®), analysing the LTE signal main performance metrics, in particular the EVM results.

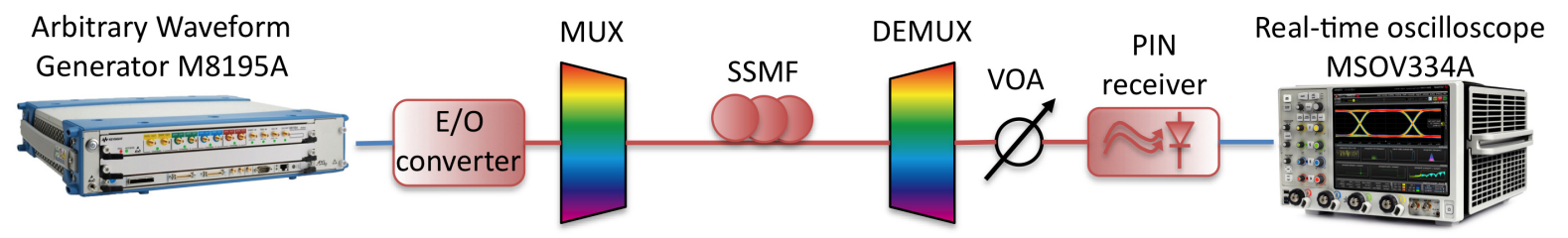

Figure 2. Optical PWM for LTE transmission experimental set up.

\section{EXPERIMENTAL RESULTS}

In order to experimentally test the proposed architecture, the PWM signals have been prepared via the SystemVue ${ }^{\circledR}$ environment. We generated LTE signals selecting different bandwidths, in particular at first we performed measurements exploiting 3-MHz LTE signals both with the RSOA based colourless transmitter in a WDM PON architecture and with a directly modulated laser. Then we transmitted a 20-MHz LTE signal employing the fiber optic PWM link. The following performance have been obtained with a transmission link of $10-\mathrm{km}$ SSMF and with overall ODN losses of $30 \mathrm{~dB}$ in both cases and network architectures. The results are obtained after PWM demodulation thanks to the vector signal analyzer testing.

\subsection{PWM transmission of 3-MHz LTE signals}

Figure 3a) shows the measured constellations for the different LTE frames; as it can be seen also from Fig. 3b), which presents a summary of the performance of the various frames, with the 3-MHz LTE it is enough to use a QPSK modulation to carry the user data (PDSCH). Figure 3c) shows the overall signal spectrum while Fig. 3d) displays the error vector spectrum, which represents, for each carrier, the difference between the EVM reference 
value and the measured one. Although the signal spectrum is almost flat, thus no apparent differences can be found between the different carriers Fig. 3d) evidences that central carriers are the most impaired. Nevertheless, measured performance highlights that the peak EVM is $6.6 \%$ and belongs to the subcarrier \#3 (anyway far below the 37\% limit for QPSK) whereas the mean EVM (among all carriers) is below $0.53 \%$ and the data EVM is $0.49 \%$.
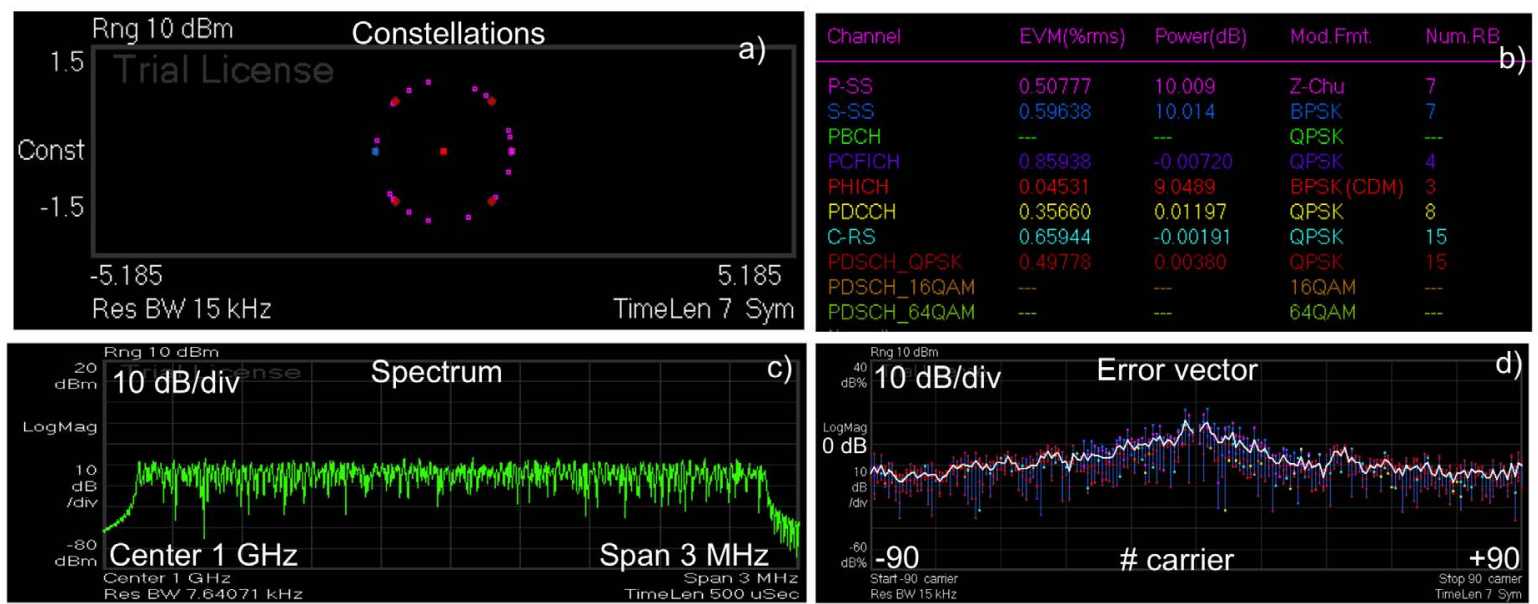

Figure 3. For the 3-MHz LTE PWM transmission: a) various constellations (up to 4 QPSK) for the different carriers (colors), b) frame EVM summary, c) spectrum of all the carriers, d) difference between the EVM reference value and the measured one vs. carrier number.

\subsection{PWM transmission of 20-MHz LTE signals}
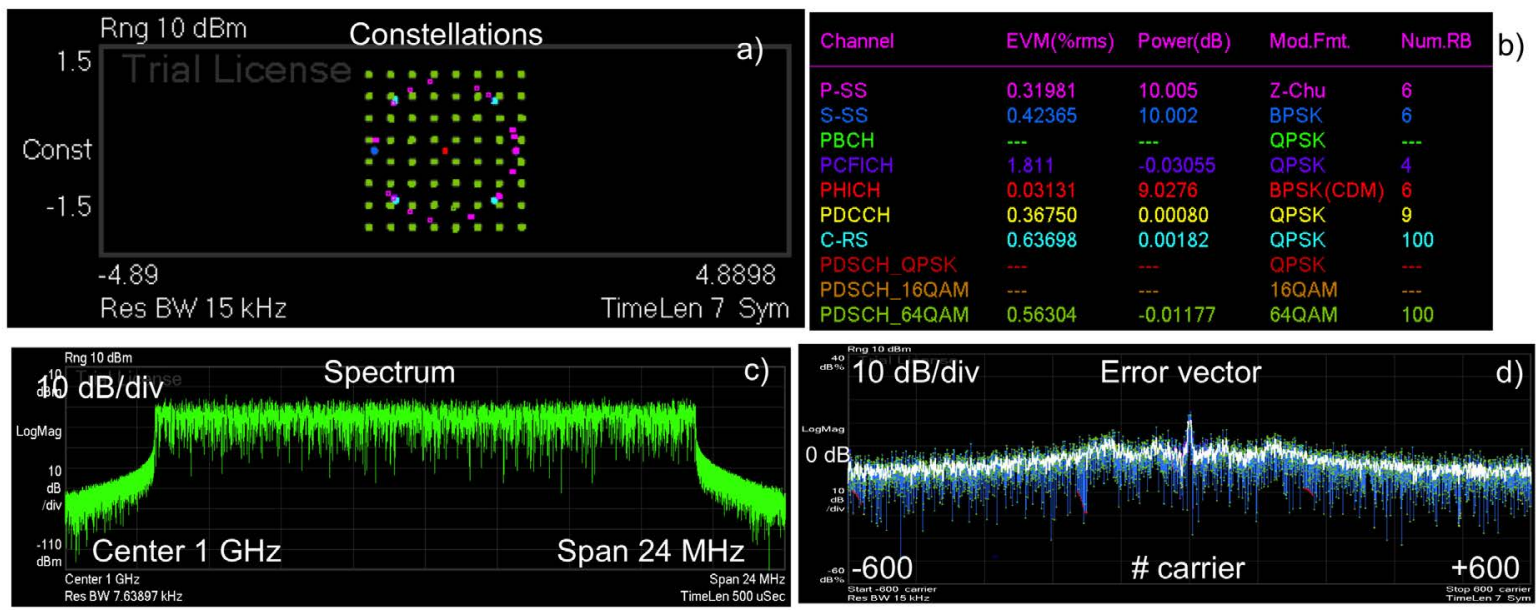

Figure 4. For the 20-MHz LTE PWM transmission: a) various constellations (up to 4 QPSK) for the different carriers (colors), b) frame EVM summary, c) spectrum of all the carriers, d) difference between the EVM reference value and the measured one vs. carrier number.

It can be seen in Fig. 4a) that in case of 20-MHz LTE signals the measured constellations present up to 64 QAM modulation. In particular, the 64 QAM is exploited to carry the user data (green points) whereas the other LTE frames support control channels with lower number of modulation levels, as evidenced in Fig. 4b) together with the performance of the different LTE frames. As for 3-MHz LTE signals Fig. 4c) shows an almost flat overall signal spectrum, which does not apparently evidence differences between the various carriers. Figure 4d), displaying the error vector spectrum, on the contrary highlights different EVM performance with respect to the reference value for the various carriers. As previously Fig. 4d) proves that central carriers are more impaired than the lateral ones in terms of error vector performance although their absolute vale EVM is not worse. In fact, measured performance demonstrates that the peak EVM is $5 \%$ and belongs to the subcarrier \#2 (anyway far below the 8\% limit for 64QAM) whereas the mean EVM (among all carriers) is below $0.53 \%$ and the data EVM is $0.56 \%$.

\section{CONCLUSIONS}

We demonstrated that optical pulse width modulation can be exploited for the transport of variable bandwidth LTE signals using directly modulated lasers or directly-modulated externally-seeded RSOAs. The exploitation of 
fibre optic links is appealing for the C-RAN fronthaul, in particular in colourless WDM PON architectures. The experimental results have been obtained after 10-km SSMF with 30-dB ODN losses and have proved that the system meets the specifications of LTE for up to 64-QAM modulation. In particular, the performance of the optical PWM transmission of LTE signals has been evaluated by SystemVue ${ }^{\circledR}$ generation and VSA ${ }^{\circledR}$ analysis that allow a thorough evaluation of the novel transmission paradigm, not only in terms of EVM.

\section{REFERENCES}

[1] A. Checko et al., "Cloud RAN for mobile networks - A technology overview," IEEE Communications Surveys and Tutorials, vol. 17, no. 1, Mar. 2015.

[2] P. Chanclou et al., "Optical fiber solution for mobile fronthaul to achieve cloud radio access network," in Proc. FNMS, Lisbon, 4 Jul. 2013, paper 9e3.

[3] P. Parolari et al., "Self-tuning transmitter for fibre-to-the-antenna PON networks," Opt. Switch. Netw., vol. 14, pp. 25-31, 2014.

[4] X. Liu et al., "Experimental demonstration of high-throughput low-latency mobile fronthaul supporting 48 20-MHz LTE signals with 59-Gb/s CPRI-equivalent rate and 2- $\mu$ s processing latency," in Proc. ECOC, Valencia, 2015, paper We 4.4.3.

[5] C. Han et al., "Linearity improvement of directly-modulated multi-IF-over-fibre LTE-A mobile fronthaul link using shunt diode predistorter," in Proc. ECOC, Valencia, 2015, paper We 4.4.4.

[6] P. Parolari, A. Gatto, L. Combi, U. Spagnolini, R. Brenot, and M. Martinelli, "Pulse-width modulation for fronthaul in a broadband-seeded RSOA WDM PON," IEEE Photon. Technol. Lett., vol. 28, pp. 1625-1628, 2016.

[7] B. Wilson and Z. Gassemlooy, "Optical fiber transmission of multiplexed video signals using pulse-width modulation," Journal of Optoelectronics, vol. 4, pp. 3-17, 1989.

[8] L. Combi, A. Gatto, M. Martinelli, P. Parolari, and U. Spagnolini, "Pulse-width optical modulation for CRAN front-hauling," in Proc. IEEE Globecom 4th International Workshop on Emerging Technologies for $5 G$ Wireless Cellular Networks, 6-10 Dec. 2015, San Diego, CA.

[9] P. Parolari, A. Gatto, L. Combi, P. Boffi, M. Martinelli, and U. Spagnolini, "Multilevel pulse width modulation fibre optic transmission for next generation mobile fronthaul," in Proc. ECOC, Dusseldorf, Sep. 2016, paper Th.2.P2.SC7.82.

[10] A. Gatto, P. Parolari, L. Combi, U. Spagnolini, R. Brenot, and M. Martinelli, "Fronthaul based on pulsewidth modulation in RSOA WDM PONs with broadband and coherent seeds," J. Opt. Commun. Netw., vol. 8, no. 11, pp. B55-B60, 2016.

[11] L. Marazzi, A. Boletti, P. Parolari, A. Gatto, R. Brenot, and M. Martinelli, "Relative intensity noise suppression in reflective SOAs," Optics Communications, vol. 318, pp. 186-188, May 2014. 